J. Korean Math. Soc. 49 (2012), No. 2, pp. 315-324

http://dx.doi.org/10.4134/JKMS.2012.49.2.315

\title{
GLOBAL EXISTENCE FOR 3D NAVIER-STOKES EQUATIONS IN A LONG PERIODIC DOMAIN
}

\author{
NAMKWON Kim AND MinkyU KWAK
}

\begin{abstract}
We consider the global existence of strong solutions of the 3D incompressible Navier-Stokes equations in a long periodic domain. We show by a simple argument that a strong solution exists globally in time when the initial velocity in $H^{1}$ and the forcing function in $L^{p}\left([0, T) ; L^{2}\right)$, $T>0,2 \leq p \leq+\infty$ satisfy a certain condition. This condition commonly appears for the global existence in thin non-periodic domains. Larger and larger initial data and forcing functions satisfy this condition as the thickness of the domain $\epsilon$ tends to zero.
\end{abstract}

\section{Introduction}

We consider the incompressible Navier-Stokes equations,

$$
\begin{array}{r}
u_{t}-\nu \triangle u+(u \cdot \nabla) u+\nabla p=f, \\
\nabla \cdot u=0,
\end{array}
$$

in a periodic domain $\Omega=T^{3}=\left[0, l_{1}\right] \times\left[0, l_{2}\right] \times\left[0, l_{3}\right]$. Here $u$ denotes the velocity of a homogeneous, viscous incompressible fluid, $f$ is the density of force per unit volume, $p$ denotes the pressure, and $\nu$ is the kinematic viscosity. We require that the forcing function $f$ and the initial data $u_{0}$ satisfy

$$
\nabla \cdot f=\nabla \cdot u_{0}=0 .
$$

We assume in addition that

$$
\int_{\Omega} f d x=\int_{\Omega} u d x=0
$$

which could be achieved by the Galilean transformation with suitable vectors $c(t)$ and $e$,

$$
u(x, t) \rightarrow u(x+c(t)+e t, t)-\frac{d c}{d t}-e .
$$

Received November 5, 2010.

2010 Mathematics Subject Classification. 35Q30, 35K51.

Key words and phrases. Navier-Stokes equations, global existence, strong solution. 
Indeed, we can take

$$
c(t)=\int_{0}^{t} \int_{0}^{r} \int f(x, s) d x d s d r, \quad e=\int u_{0} d x .
$$

By the classical results of Leray and Hopf ([11], [4]), there exists a global weak solution of the Navier-Stokes equations in a three dimensional torus. It is also known that the solution becomes necessarily strong (regular) for all regular data in a two dimensional domain. But in a three dimensional domain, global strong solutions have only been guaranteed for small initial data (See, for example, [2], [3], [14], [15] and the references therein).

In [13], Raugel and Sell treated the problem on thin periodic domain, $\Omega=$ $\left(0, l_{1}\right] \times\left(0, l_{2}\right] \times(0, \epsilon]$ and they obtained a significant existence result on global regular solutions. The main idea is that if the thickness of the domain is small enough, the solution of the Navier-Stokes equations is close to the 2D NavierStokes equations. They proved that there are large sets $R(\epsilon) \subset H^{1}(\Omega)$ and $S(\epsilon) \subset L^{\infty}\left((0, \infty), L^{2}(\Omega)\right)$ such that if $u(0)=u_{0} \in R(\epsilon)$ and $f \in S(\epsilon)$, then there exists a strong solution $u(t)$ that remains in $H^{1}(\Omega)$ for all $t \geq 0$. The sets $R(\epsilon)$ and $S(\epsilon)$ get larger and larger as $\epsilon \rightarrow 0$.

Since then, there have been many improvements on the estimates of the size of these sets $R(\epsilon)$ and $S(\epsilon)$ under various boundary conditions (See [1], [5], $[12],[6],[7],[8],[9],[16]$ and the references therein). Roughly, under various boundary conditions except the periodic boundary condition, it has been shown that if

$$
\left\|u_{0}\right\|_{H^{1}} \leq C \epsilon^{-1 / 2} \quad \text { and } \quad\|f\|_{L^{\infty}\left((0, \infty), L^{2}\right)} \leq C \epsilon^{-1 / 2}
$$

for some constant $C=C(\nu)$, then the corresponding global strong solution exists (See [1], [16]). We note that the above condition can cover very large initial data and forcing functions if $\epsilon>0$ is small enough.

However, under the periodic boundary condition, it is not known whether (4) implies the existence of global strong solutions. Until now, it is known that, when $f=0$, the existence of the global strong solution is guaranteed under the condition $([10])$

$$
\left\|u_{0}\right\|_{H^{1}} \leq C \epsilon^{-1 / 2}|\log \epsilon|^{1 / 2},
$$

or under the following condition $([6])$

$$
\begin{aligned}
& \left\|\left(N u_{0}\right)_{3}\right\| \leq C \nu \epsilon^{1 / 2},\|N f\|_{L^{\infty}\left(0, \infty ; L^{2}\right)} \leq C \nu^{2} \epsilon^{1 / 2}, \\
& \left\|\nabla u_{0}\right\| \leq C \nu \epsilon^{-1 / 2},\|f\|_{L^{\infty}\left(0, \infty ; L^{2}\right)} \leq C \nu^{2} \epsilon^{-1 / 2} .
\end{aligned}
$$

Here, $N$ is the average operator with respect to the thin direction. We note that the first two conditions in the above are not so restrictive since $N u_{0}$ and $N f$ are independent of the third variable and so they are in fact $\epsilon$ independent conditions.

In this paper, we consider the global existence of strong solutions in a long periodic domain, $\Omega=(0, \epsilon] \times(0, \epsilon] \times(0, l]$. We first prove in a simple way that 
a global strong solution exists whenever the initial and the forcing functions satisfy for any $2 \leq p \leq \infty$ and $L>0$,

$$
\left\|\nabla u_{0}\right\|_{L^{2}} \leq \frac{C \nu}{L} \quad \text { and } \quad\|f\|_{L^{p}\left((0, \infty), L^{2}\right)} \leq C \nu^{(2 p-1) / p} \lambda_{1}^{(3 p-4) / 4 p}
$$

together with a mild condition,

$$
\frac{1}{L}\left\|u_{0}\right\|_{L^{2}} \leq 1
$$

for some universal constant $C$. Here, $\lambda_{1}=4 \pi^{2} / l$ is the first eigenvalue of the Stokes operator. This result is obtained simply by considering a differential inequality for a product of norms, which is comparable to $H^{1 / 2}$ norm. The most natural choice of $L$ in the condition (6) is $L=\sqrt{|\Omega|}$, which is not practically restrictive since it just means that the spatial average of the square of the velocity is bounded by a suitable constant. Then, when the domain is long rod type $\Omega=(0, \epsilon] \times(0, \epsilon] \times(0, l]$, the choice $L=\sqrt{|\Omega|}$ becomes of order $\epsilon$ and the bound on $H^{1}$ norm of the velocity in (5) is improved greatly compared to the case of thin domain. We also give a condition independent of the $L^{2}$ norm of the velocity. Concretely, we show that the global regularity is guaranteed if

$$
\left\|\nabla u_{0}\right\| \leq C \nu \epsilon^{-1 / 2},\|f\|_{p, 2} \leq C \nu^{(2 p-1) / p} \epsilon^{-1 / 2}
$$

for any $2 \leq p \leq \infty$. The above condition exactly recovers (4) even for more general $p$ and supports that the condition (4) might be enough for the global existence in a thin periodic domain under the periodic boundary condition.

This work has been partially done while the first author is visiting the University of Minnesota for a sabbatical year and he is grateful to the School of Mathematics for their warm hospitalities and specially to Prof. G. R. Sell, Vladimir Sverak and Luan Hoang.

\section{Preliminary estimates}

Throughout the paper, $\Omega=(0, \epsilon] \times(0, \epsilon] \times(0, l]$. Here, $l$ is a fixed constant and $\epsilon>0$ is a small parameter. For convenience's sake, we denote the two dimensional torus $D \equiv D_{\epsilon}=(0, \epsilon] \times(0, \epsilon]$. The function spaces we work with are

$$
H=\left\{u \in L^{2}(\Omega) \mid \nabla \cdot u=0, \int_{\Omega} u=0\right\}
$$

and $V=H \cap W^{1,2}(\Omega)$. It is well known that $\|\nabla u\|_{L^{2}}$ is an equivalent norm for $V$ due to the Poincaré inequality. For convenience's sake, we also denote

$$
\|\cdot\|_{L^{p}}=\|\cdot\|_{p}, \quad\|\cdot\|_{2}=\|\cdot\|, \quad\|\cdot\|_{L^{p}\left(0, \infty ; L^{q}(\Omega)\right)}=\|\cdot\|_{p, q},
$$

the Leray projection on $L^{2}(\Omega)$ into $H$ by $\mathbb{P}$, and the Stokes operator by $A=$ $\mathbb{P}(-\Delta)$. We define the bilinear form $B(u, v)=\mathbb{P}(u \cdot \nabla) v$ and the trilinear form $b(u, v, w)$ by

$$
b(u, v, w)=\langle B(u, v), w\rangle=\int_{\Omega} B(u, v) \cdot w d x
$$


We now define an orthogonal projection $M$ on $L^{2}\left(\Omega_{\epsilon}\right)$ by

$$
M u=\frac{1}{\epsilon^{2}} \int_{0}^{\epsilon} \int_{0}^{\epsilon} u\left(x_{1}, x_{2}, x_{3}\right) d x_{1} d x_{2}
$$

and denote $v \equiv M u$ and $w \equiv(I-M) u$ for simplicity. Note that the above projection is different from the one in [6]. Here, $v=v\left(x_{3}\right)$ and $\nabla \cdot v=0$. So, $v_{3}$ must be a constant in space. Since we assume (3) from the first, we then get

$$
v_{3}=\frac{1}{|\Omega|} \int v_{3}=\frac{1}{|\Omega|} \int u_{3}=0 .
$$

It is clear that the following Poincaré inequality holds for $w \in H^{1}$ since $M w=$ 0 :

$$
\|w\|^{2} \leq C \epsilon^{2}\|\nabla w\|^{2} .
$$

Further, $w$ satisfies the following inequalities, which are basically GargliardoNirenberg inequalities.

Lemma 2.1. Given $u \in V \cap D(A)$, let $v=M u$ and $w=(I-M) u$. We have

$$
\begin{gathered}
\|\nabla v\|_{\infty} \leq \frac{C}{\epsilon}\|\nabla v\|^{1 / 2}\|A v\|^{1 / 2}, \\
\|\nabla w\|_{q} \leq C\left(\left\|\nabla_{3} w\right\|\|w\|+\|w\|^{2}\right)^{1 / 2 q}\|A w\|^{\frac{q-1}{q}}, \quad 1<q \leq 3 .
\end{gathered}
$$

Here, all $C$ 's are independent of $\epsilon$.

Proof. Since $w\left(\cdot, x_{3}\right)$ is average zero on $D$ for any $x_{3} \in(0, l], w$ satisfies the following two dimensional Gargliardo-Nirenberg inequality.

$$
\|\nabla w\|_{L^{q}(D)}^{q} \leq C\left\|\nabla^{2} w\right\|_{L^{2}(D)}^{q-1}\|w\|_{L^{2}(D)} .
$$

Here, $C$ is independent from $\epsilon$. In fact, the above inequality is scaling invariant. Integrating with respect to $x_{3}$, we have

$$
\int_{0}^{l} d x_{3} \int_{D}|\nabla w|^{q} \leq C \int_{0}^{l} d x_{3}\left(\int_{D}\left|\nabla^{2} w\right|^{2}\right)^{\frac{q-1}{2}} \sup _{x_{3}}\|w\|_{L^{2}(D)}\left(x_{3}\right) .
$$

While,

$$
\begin{aligned}
\|w\|_{L^{2}(D)}^{2}(b) & \leq\left|\int_{a}^{b} d x_{3} \partial_{3}\|w\|_{L^{2}(D)}^{2}\left(x_{3}\right)\right|+\|w\|_{L^{2}(D)}^{2}(a) \\
& \leq \int_{a}^{b} \int_{D}\left|\partial_{3} w\|w \mid d x+\| w \|_{L^{2}(D)}^{2}(a)\right. \\
& \leq\left\|\nabla_{3} w\right\|\|w\|+\|w\|_{L^{2}(D)}^{2}(a)
\end{aligned}
$$

Integrating the above with respect to $a$ over $(0, l]$, we have

$$
\sup _{x_{3}}\|w\|_{L^{2}(D)}^{2} \leq\left\|\nabla_{3} w\right\|\|w\|+\frac{1}{l}\|w\|^{2} .
$$


Plugging the above into (12) and using the Hölder inequality, we have

$$
\int_{\Omega}|\nabla w|^{q} \leq C\left\|\nabla^{2} w\right\|^{q-1}\left(\left\|\nabla_{3} w\right\|\|w\|+\|w\|^{2}\right)^{1 / 2}
$$

Since $\left\|\nabla^{2} w\right\| \leq C\|A w\|$, we have the desired inequality (11). Similarly,

$$
\left(\partial_{3} v_{i}\right)^{2}(b)=2 \int_{a}^{b} d x_{3} \partial_{3}^{2} v_{i} \partial_{3} v_{i}+\left(\partial_{3} v\right)^{2}(a) .
$$

There exists $a$ such that $\partial_{3} v_{i}(a)=0$ since $\partial_{3} v_{i}$ is average zero. Thus we have

$$
\left(\nabla v_{i}\right)^{2}(b) \leq C \int d x_{3}\left|\nabla^{2} v\left\|\nabla v \mid \leq \frac{C}{\epsilon^{2}}\right\| \nabla v\|\| A v \|\right.
$$

Taking supremum with respect to $b$ and adding them up for $i=1,2$, we have the desired result (10).

We now present the following estimates concerning the trilinear form $b$. We use the above lemma with $q=3$ to get the estimates.

Lemma 2.2. Let $v$ and $w$ be as before, we have

$$
\begin{gathered}
|b(w, w, A w)| \leq C\|w\|^{1 / 2}\|\nabla w\|^{1 / 2}\|A w\|^{2} \\
|b(v, w, A w)|,|b(w, v, A w)|,|b(w, w, A v)| \\
\leq C\|\nabla v\|^{1 / 2}\|A v\|^{1 / 2}\|w\|^{1 / 2}\|A w\|^{3 / 2} .
\end{gathered}
$$

Here, all $C$ 's are independent from $\epsilon$.

Proof. First, by integration by parts,

$$
\begin{aligned}
b(w, w, A w) & =-\int(w \cdot \nabla) w \cdot \Delta w=\int\left(\nabla_{j} w \cdot \nabla\right) w \cdot \nabla_{j} w+w \cdot \nabla\left(\nabla_{j} w\right) \nabla_{j} w \\
& =\int\left(\nabla_{j} w \cdot \nabla\right) w \cdot \nabla_{j} w .
\end{aligned}
$$

Thus, using (11) with $q=3,(9)$, and the smallness of $\epsilon$,

$$
\begin{aligned}
|b(w, w, A w)| \leq C\|\nabla w\|_{3}^{3} & \leq C\|A w\|^{2}\left(\left\|\nabla_{3} w\right\|^{1 / 2}\|w\|^{1 / 2}+\|w\|\right) \\
& \leq C\|A w\|^{2}\|\nabla w\|^{1 / 2}\|w\|^{1 / 2} .
\end{aligned}
$$

By similar argument,

$$
b(v, w, A w)=\int\left(\nabla_{j} v \cdot \nabla\right) w \cdot \nabla_{j} w .
$$

Then, since $v$ depends only on $x_{3}$,

$$
\begin{aligned}
|b(v, w, A w)| & \leq \int_{0}^{l} d x_{3}|\nabla v| \int_{D}|\nabla w|^{2} \\
& \leq C\|\nabla v\|_{L^{\infty}(0, l)}\|\nabla w\|^{2} \leq C\|\nabla v\|^{1 / 2}\|A v\|^{1 / 2}\|\nabla w\|\|A w\| \\
& \leq C\|\nabla v\|^{1 / 2}\|A v\|^{1 / 2}\|w\|^{1 / 2}\|A w\|^{3 / 2}
\end{aligned}
$$


Here, we used in the last line the interpolation inequality

$$
\|\nabla f\|^{2}=-\int f \Delta f \leq\|f\|\|A f\| .
$$

Similarly,

$$
\begin{aligned}
|b(w, v, A w)| & \leq \int_{0}^{l} d x_{3}|\nabla v|\|w\|_{L^{2}(D)}\|A w\|_{L^{2}(D)} \\
& \leq C\|\nabla v\|_{L^{\infty}(0, l)}\|w\|\|A w\| \leq C\|\nabla v\|^{1 / 2}\|A v\|^{1 / 2}\|w\|\|A w\|^{3 / 2} \\
|b(w, w, A v)| & =\left|\int \nabla_{j} w \cdot \nabla w \cdot \nabla_{j} v+w \cdot \nabla \nabla_{j} w \cdot \nabla_{j} v\right| \\
& \leq C\|\nabla v\|^{1 / 2}\|A v\|^{1 / 2}\|w\|^{1 / 2}\|A w\|^{3 / 2}
\end{aligned}
$$

\section{Regularity}

In this section, we give our main result. We first reformulate (1)-(2) in the standard nonlinear evolutionary equation on the Hilbert space $V$,

$$
u_{t}+\nu A u+B(u, u)=\mathbb{P} f \text {. }
$$

We shall consider solutions of (16) with the initial data $u_{0}$ and $f=f(t)$ in the class

$$
u_{0} \in V, \quad f(t) \in L^{p}([0, \infty), H), p \geq 2 .
$$

We first present the following theorem, which is simple and shows the underlying idea of our result.

Theorem 3.1. Given any $p \geq 2$, the Navier-Stokes evolutionary equation (16) has a solution

$$
u \in C^{0}([0, \infty), H) \cap L^{\infty}((0, \infty), V)
$$

if

$$
\left\|u_{0}\right\|\left\|\nabla u_{0}\right\|+2 \nu^{-\frac{2 p-2}{p}} \lambda_{1}^{-\frac{3 p-4}{2 p}}\|f\|_{p, 2}^{2} \leq \frac{\nu^{2}}{C^{2}} .
$$

Here, $\lambda_{1}$ is the first eigenvalue of the Stokes operator, $C$ is an absolute constant independent of $\epsilon$. Moreover, in this case

$$
\|\nabla u\|^{2}(t) \leq\left\|\nabla u_{0}\right\|^{2}+4 \nu^{-\frac{2 p-2}{p}} \lambda_{1}^{-\frac{p-2}{p}}\|f\|_{p, 2}^{2}
$$

for all $t>0$.

Proof. By taking the scalar product of (16) with $u$ and using the fact that

$$
\int B(u, u) u d x=0
$$

we find that

$$
\frac{d}{d t}\|u\|^{2}+2 \nu\|\nabla u\|^{2} \leq 2\|f\|\|u\|
$$


Since $v$ depends only on $x_{3}$ and $v_{3}=0,\langle B(v, v), A v\rangle=0$ and $b(v, v, w)=$ $b(w, v, v)=b(v, w, v)=0$. So,

$$
\begin{aligned}
\langle B(u, u), A u\rangle= & \langle B(v, w), A w\rangle+\langle B(w, v), A w\rangle \\
& +\langle B(w, w), A v\rangle+\langle B(w, w), A w\rangle \\
\leq & C\left(\|\nabla v\|^{1 / 2}\|A v\|^{1 / 2}\|w\|^{1 / 2}\|A w\|^{3 / 2}+\|w\|^{1 / 2}\|\nabla w\|^{1 / 2}\|A w\|^{2}\right) \\
\leq & C\|w\|^{1 / 2}\|\nabla u\|^{1 / 2}\|A u\|^{2}
\end{aligned}
$$

by the orthogonality of $v$ and $w$. Then, taking the scalar product of (16) with $A u$ and using the above estimate, we obtain

$$
\begin{aligned}
\frac{d}{d t}\|\nabla u\|^{2}+2 \nu\|A u\|^{2} & \leq 2\left|\int f A u\right|+\left|\int B(u, u) A u\right| \\
& \leq 2\|f\|\|A u\|+C(\|w\|\|\nabla u\|)^{1 / 2}\|A u\|^{2} .
\end{aligned}
$$

Now, we multiply (20) by $\|\nabla u\|^{2}$ and (21) by $\|u\|^{2}$ and adding them to have

$$
\begin{gathered}
\frac{d}{d t}\left(\|u\|^{2}\|\nabla u\|^{2}\right)+2 \nu\|\nabla u\|^{4}+2 \nu\|u\|^{2}\|A u\|^{2} \\
(22) \leq 2\|f\|\|u\|\left(\|\nabla u\|^{2}+\|u\|\|A u\|\right)+C(\|u\|\|\nabla u\|)^{1 / 2}\|u\|^{2}\|A u\|^{2} .
\end{gathered}
$$

By the Young inequality and (15), we have

$$
\begin{aligned}
2\|f\|\|u\|\left(\|\nabla u\|^{2}+\|u\|\|A u\|\right) & \leq 4\|f\|\|u\|^{2}\|A u\| \\
& \leq 4\|f\| \frac{\|\nabla u\|^{1 / 2}}{\lambda_{1}^{1 / 4}}\|u\|^{1 / 2}\|u\|\|A u\| \\
& \leq \nu\|u\|^{2}\|A u\|^{2}+\frac{4}{\nu \lambda_{1}^{1 / 2}}\|f\|^{2}\|u\|\|\nabla u\| .
\end{aligned}
$$

Denoting $G^{2}=\|u\|^{2}\|\nabla u\|^{2}$, we thus arrive at

$$
\frac{d}{d t} G^{2}+\nu \lambda_{1} G^{2} \leq\left[C G^{1 / 2}-\nu\right]\|u\|^{2}\|A u\|^{2}+\frac{4}{\nu \lambda_{1}^{1 / 2}}\|f\|^{2} G .
$$

If $G(t) \leq \frac{\nu^{2}}{C^{2}}$ for all $t>0$,

$$
\frac{d}{d t} G+\nu \lambda_{1} G \leq \frac{2}{\nu \lambda_{1}^{1 / 2}}\|f\|^{2}
$$

Therefore, by the Grönwall inequality,

$$
\begin{aligned}
G & \leq G(0) e^{-\nu \lambda_{1} t}+\frac{2}{\nu \lambda_{1}^{1 / 2}} \int_{0}^{t}\|f\|^{2}(s) e^{\nu \lambda_{1}(s-t)} d s \\
& \leq G(0)+\frac{2}{\nu \lambda_{1}^{1 / 2}}\|f\|_{p, 2}^{2}\left(\frac{p-2}{p \nu \lambda_{1}}\right)^{(p-2) / p} \\
& \leq G(0)+2\left(\frac{p-2}{p}\right)^{\frac{p-2}{p}} \nu^{-\frac{2 p-2}{p}} \lambda_{1}^{-\frac{3 p-4}{2 p}}\|f\|_{p, 2}^{2}
\end{aligned}
$$


for any $p \geq 2$. Note that the above estimate holds true even for $p=2$ and $\infty$. Since $((p-2) / p)^{(p-2) / p} \leq 1$, the typical continuation argument and (18) justifies the above argument and we indeed have $G(t) \leq \frac{\nu^{2}}{C^{2}}$ for all $t>0$. Furthermore, if $G(t) \leq \frac{\nu^{2}}{C^{2}}$, we apply the Hölder inequality to (21) to have

$$
\frac{d}{d t}\|\nabla u\|^{2}+\frac{\nu}{2} \lambda_{1}\|\nabla u\|^{2} \leq \frac{2}{\nu}\|f\|^{2} .
$$

Again, by the Grönwall inequality,

$$
\|\nabla u\|^{2}(t) \leq\left\|\nabla u_{0}\right\|^{2}+\int_{0}^{t} \frac{2}{\nu}\|f\|^{2}(s) e^{\nu \lambda_{1}(s-t) / 2} d s,
$$

which gives (19) and finishes the proof.

The condition (18) is in a sense a condition of smallness of the initial data and external force. However, this condition allows for initial data with large $H^{1}$ norm provided that the $L^{2}$ norm of the initial data and $f$ are small enough. In particular, when $f=0$, the above theorem tells that there exists a globally regular solution if $\left\|u_{0}\right\|$ is small enough compared with $\nu^{2}\left\|\nabla u_{0}\right\|^{-1}$. As a corollary of the above theorem, we have the following.

Corollary 3.2. There exists a globally regular solution if initial data satisfies (5) and (6) with $L=\epsilon$ when $\Omega=[0, l] \times[0, \epsilon] \times[0, \epsilon]$.

Applying the projections $M$ and $(I-M)$ to the equation (16) and using $M B(v, v)=B(v, v)=0$ and $M B(v, w)=M B(w, v)=0$, we get the equation for $v$,

$$
\frac{d v}{d t}+\nu A v=M f-M B(w, w)
$$

and the equation for $w$,

$$
\frac{d w}{d t}+\nu A w=(I-M) f-B(w, v)-B(v, w)-(I-M) B(w, w) .
$$

Theorem 3.3. There exists a globally regular solution $u$ of (16) in $\Omega$ if, for some constant $C$,

$$
\left\|\nabla u_{0}\right\|^{2}+2 \nu^{-\frac{2 p-2}{p}} \lambda_{1}^{-\frac{p-2}{p}}\|f\|_{p, 2}^{2}<\frac{\nu^{2}}{4 C^{2}} \epsilon^{-1} .
$$

Proof. We start from (21). By (9), (21) becomes

$$
\frac{d}{d t}\|\nabla u\|^{2}+2 \nu\|A u\|^{2} \leq 2\|f\|\|A u\|+C(\epsilon\|\nabla w\|\|\nabla u\|)^{1 / 2}\|A u\|^{2} .
$$

Then,

$$
\frac{d}{d t}\|\nabla u\|^{2}+\left(\nu-C \epsilon^{1 / 2}\|\nabla u\|\right)\|A u\|^{2} \leq \frac{1}{\nu}\|f\|^{2}
$$

since $\|\nabla u\| \geq\|\nabla w\|$. Now, we apply the Grönwall lemma to the above inequality with typical smallness argument. That is, since $\left\|\nabla u_{0}\right\|<\frac{\nu}{2 C} \epsilon^{-1 / 2}$ 
from (25), if $\|\nabla u\|(t)>\frac{\nu}{2 C} \epsilon^{-1 / 2}$ for some $t>0$, there would be the first time $t=T$ such that $\|\nabla u\|(T)=\frac{\nu}{2 C} \epsilon^{-1 / 2}$. However, for $0<t<T$,

$$
\frac{d}{d t}\|\nabla u\|^{2}+\frac{\nu}{2} \lambda_{1}\|\nabla u\|^{2}<\frac{1}{\nu}\|f\|^{2}
$$

Applying the Grönwall lemma to the above inequality, we would have

$$
\begin{aligned}
\|\nabla u\|^{2}(T) & <\left\|\nabla u_{0}\right\|^{2}+\frac{1}{\nu} \int_{0}^{T}\|f\|^{2} e^{\nu \lambda_{1}(s-T) / 2} d s \\
& \leq\left\|\nabla u_{0}\right\|^{2}+\frac{1}{\nu}\left(\frac{2(p-2)}{\nu \lambda_{1} p}\right)^{(p-2) / p}\|f\|_{p, 2}^{2} \\
& \leq\left\|\nabla u_{0}\right\|^{2}+2 \nu^{-\frac{2 p-2}{p}} \lambda_{1}^{-\frac{p-2}{p}}\|f\|_{p, 2}^{2} .
\end{aligned}
$$

If (25) holds true, this leads a contraction. Therefore, $\|\nabla u\|^{2}<\frac{\nu^{2}}{4 C^{2}} \epsilon^{-1}$ for all $t>0$ and we finish the proof.

Clearly, the condition (25) in particular implies that there exists a globally regular solution if, for suitable $C$,

$$
\left\|\nabla u_{0}\right\| \leq C \nu \epsilon^{-1 / 2},\|f\|_{p, 2} \leq C \nu^{(2 p-1) / p} \epsilon^{-1 / 2}
$$

since $\lambda_{1}=\frac{4 \pi^{2}}{l^{2}}$ is fixed.

Acknowledgements. The first author was supported by the Korea Research Foundation Grant funded by the Korean Government(MOEHRD, Basic Research Promotion Fund)(KRF-2007-521-C00021) and the second author was supported in part by KRF 2009-0074605.

\section{References}

[1] J. D. Avrin, Large-eigenvalue global existence and regularity results for the NavierStokes equations, J. Differential Equations 127 (1996), no. 2, 365-390.

[2] P. Constantin and C. Foias, Navier-Stokes Equations, University of Chicago Press, Chicago, 1988.

[3] H. Fujita and T. Kato, On the Navier-Stokes initial value problem, Arch. Rational Mech. Anal. 16 (1964), 269-315.

[4] E. Hopf, Über die Anfangswertaufgabe fur die hydrodynamischen Grudgleichungen, Math. Nachr. 4 (1951), 213-231.

[5] D. Iftimie, The 3D Navier-Stokes equations seen as a perturbation of the 2D NavierStokes equations, Bull. Soc. Math. France 127 (1999), no. 4, 473-517.

[6] D. Iftimie and G. Raugel, Some results on the Navier-Stokes equations in thin 3D domains, J. Differential Equations 169 (2001), no. 2, 281-331.

[7] D. Iftimie, G. Raugel, and G. R. Sell, Navier-Stokes equations in thin 3D domains with Navier boundary conditions, Indiana Univ. Math. J. 56 (2007), no. 3, 1083-1156.

[8] M. Kwak and N. Kim, Remark on global existence for 3D Navier-Stokes equations in Lipschitz domain, Submitted (2007).

[9] I. Kukavica and M. Ziane, Regularity of the Navier-Stokes equation in a thin periodic domain with large data, Discrete Contin. Dyn. Syst. 16 (2006), no. 1, 67-86.

[10] _ On the regularity of the Navier-Stokes equation in a thin periodic domain, J. Differential Equations 234 (2007), no. 2, 485-506. 
[11] J. Leray, Sur le mouvement d'un liquide visqueux emplissant l'espace, Acta Math. 63 (1934), no. 1, 193-248.

[12] S. Mongtgomery-Smith, Global regularity of the Navier-Stokes equations on thin three dimensional domains with periodic boundary conditions, Electron. J. Differential Equations 1999 (1999), no. 11, 1-19.

[13] G. Raugel and G. R. Sell, Navier-Stokes equations on thin 3D domains. I. Global attractors and global regularity of solutions, J. Amer. Math. Soc. 6 (1993), no. 3, 503-568.

[14] G. R. Sell and Y. You, Dynamics of Evolutionary Equations, Applied Math. Sciences 143, Springer, Berlin, 2002.

[15] R. Temam, Navier-Stokes Equations and Nonlinear Functional Analysis, CBMS Regional Conference Series, No. 66, SIAM, Philadelphia, 1995.

[16] R. Temam and M. Ziane, Navier-Stokes equations in three-dimensional thin domains with various boundary conditions, Adv. Differential Equations 1 (1996), no. 4, 499-546.

NAMKWON KIM

Department of Mathematics

Chosun University

KwangJu 501-759, Korea

E-mail address: kimnamkw@chosun.ac.kr

MINKYU KWAK

Department of Mathematics

Chonnam National University

KwanguU 500-757, Korea

E-mail address: mkkwak@jnu.ac.kr 